\title{
Probe set filtering increases correlation between Affymetrix GeneChip and qRT-PCR expression measurements
}

\author{
Jakub Mieczkowski ${ }^{1 *}$, Magdalena E Tyburczy ${ }^{1}$, Michal Dabrowski ${ }^{1}$, Piotr Pokarowski ${ }^{2 *}$
}

\begin{abstract}
Background: Affymetrix GeneChip microarrays are popular platforms for expression profiling in two types of studies: detection of differential expression computed by $p$-values of $t$-test and estimation of fold change between analyzed groups. There are many different preprocessing algorithms for summarizing Affymetrix data. The main goal of these methods is to remove effects of non-specific hybridization, and to optimally combine information from multiple probes annotated to the same transcript. The methods are benchmarked by comparison with reference methods, such as quantitative reverse-transcription PCR (qRT-PCR).

Results: We present a comprehensive analysis of agreement between Affymetrix GeneChip and qRT-PCR results. We analyzed the influence of filtering by fraction Present calls introduced by J.N. McClintick and H.J. Edenberg (2006) and 2 mapping procedures: updated probe sets definitions proposed by Dai et al. (2005) and our "naive mapping" method. Because of evolution of genome sequence annotations since the time when microarrays were designed, we also studied the effect of the annotation release date. These comparisons were prepared for 6 popular preprocessing algorithms (MAS5, PLIER, RMA, GC-RMA, MBEI, and MBEImm) in the 2 above-mentioned types of studies. We used data sets from 6 independent biological experiments. As a measure of reproducibility of microarray and qRT-PCR values, we used linear and rank correlation coefficients.

Conclusions: We show that filtering by fraction Present calls increased correlations for all 6 preprocessing algorithms. We observed the difference in performance of PM-MM and PM-only methods: using MM probes increased correlations in fold change studies, but PM-only methods proved to perform better in detection of differential expression. We recommend using GC-RMA for detection of differential expression and PLIER for estimation of fold change. The use of the more recent annotation improves the results in both types of studies, encouraging re-analysis of old data.
\end{abstract}

\section{Background}

Affymetrix GeneChip microarrays (MA) are routinely used for genome-wide quantitative expression analysis. MA measure the expression of genes using probe sets. Probe sets are composed of multiple probes covering different regions of a particular transcript (PerfectMatch, PM), as well as probes designed to measure non-specific hybridization (Mismatch, MM).

\footnotetext{
* Correspondence: j.mieczkowski@nencki.gov.pl; pokar@mimuw.edu.pl 'Laboratory of Transcription Regulation, Department of Cell Biology, The Nencki Institute of Experimental Biology, Pasteur 3, 02-093 Warsaw, Poland ${ }^{2}$ Institute of Informatics, Faculty of Mathematics, Informatics and Mechanics, Banacha 2, University of Warsaw, 02-097 Warsaw, Poland
}

Preprocessing algorithms evaluate the signals from probes and combine them to get an expression measure for each probe set. As was shown in [1-4], the choice of the preprocessing algorithm has a strong impact on MA analysis. Irizarry et al. [5] presented a comprehensive comparison of such algorithms and concluded that different preprocessing algorithms may suit different applications. In this study, we analyzed 6 popular algorithms: MAS5 [6], PLIER [7], RMA [8], GC-RMA [9], and 2 variants of MBEI algorithm [10,11], using either PM-only model (MBEI) or PM-MM model (MBEImm).

Quantitative reverse-transcription PCR (qRT-PCR) is another method to measure gene expression. This is a widely used diagnostic assay, but the measurements

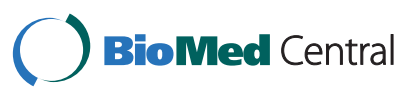


obtained with this technique are more costly and time consuming (per gene) than in MA analysis. In biological studies [12], as well as in analyses of preprocessing algorithms [13], qRT-PCR is widely used to validate MA results. As was shown in [3], the correlation coefficient between log-transformed $\left(\log _{2}\right)$ microarray expression measurements and $\Delta C_{T}$ values from qRT-PCR is a good measure of agreement.

Depending on the type, MA consist of several or tens of thousands probe sets, and traditional analysis of microarray data requires testing of all possible probe sets. However, multiple testing increases the number of false positives. For this reason, methods that eliminate unreliable measurements are helpful. One of them is filtering by fraction Present calls $(F)$ which was proposed by McClintick and Edenberg [14] (for details see the Methods section). This method uses the detection call (Present/Absent/Marginal or P/A/M) originally designed to support the Affymetrix Microarray Suite 5 (MAS5), one of the first preprocessing algorithms. The $\mathrm{P} / \mathrm{A} / \mathrm{M}$ procedure uses both $\mathrm{PM}$ and MM probes to determine specificity of signal of each probe set. The filtering by fraction Present calls removes probe sets that had the fraction of Present calls below a given threshold in every sub-group of samples. The authors showed that $F$ can be effectively applied even for data generated by another preprocessing algorithm. Recently, Hackstadt and Hess [15] compared different filtering methods on 3 biological data sets and pointed that $F$ can increase the number of differentially expressed genes identified.

As was shown by Okoniewski et al. [16], there are many probe sets which are annotated ambiguously to several genes, so cross-hybridization signal might be seen. The authors demonstrated that such probe sets could hamper inference of gene interaction networks, and suggested the use of a comprehensive annotation of probe sets to genes. An example of such mapping are custom definition files packages (CDF packages denoted in our work as D), designed by Dai et al. [17]. Their redefinitions of probe sets are based on the latest genomic knowledge, and by regrouping probes and creating new probe sets, allow to limit analysis only to the genespecific measurements. However, among the redefined probe sets the numbers of probes are not identical. Since such disparity may cause different quality of results, we implemented "naive mapping" method $(N)$ which reduces the set of analyzed probe sets only to probe sets annotated to a single gene (for details see the Methods section). In our study of filtering/mapping effects, we compared 6 approaches: $A$ - use of all probe sets without any filtering or mapping method, $F, D, N$ as described above, and all their nontrivial combinations - FD and FN.
Both $D$ and $N$ mappings demand an annotation of probe sets to genes, and there are many different databases or probe set definitions for this purpose. Sandberg and Larsson [18] compared the accuracy in estimation of fold change computed with MA for 6 databases: NetAffx (original), ENSEMBL gene, ENSEMBL transcript, Entrez, RefSeq, and UniGene. Accuracy was defined as a slope after a linear regression between qRT-PCR and MA fold change for 16 genes. The authors observed the most significant improvement in accuracy when using the ENSEMBL gene and transcript databases (Figure 2 in [18]). Since qRT-PCR data used in our comparisons are labelled with the gene rather than the transcript names, we decided to use the ENSEMBL gene database.

Because of the evolution of genome sequence annotations, probe sets that originally were mapped uniquely to one gene may now be mapped to several genes. Therefore, to check the influence of this evolution of annotation, we decided to use 2 different versions of annotations: the new (ENSEMBL version 55 issued in Jul 2009 and custom CDF version 12 issued in Jul 2009) and the old (ENSEMBL version 49 issued in Mar 2008 and custom CDF version 11 issued in Nov 2008).

Typically, MA experiments are designed to profile the expression in two types of study: detection of differentially expressed genes $[1,3,19]$ or estimation of fold change [13]. The difference of expression is usually measured by p-values of Welch's $t$-statistic that can be calculated as follows:

$$
t=\frac{\bar{X}_{1}-\bar{X}_{2}}{\sqrt{\frac{s_{1}^{2}}{n_{1}}+\frac{s_{2}^{2}}{n_{12}}}},
$$

where $\bar{X}_{1}$ and $\bar{X}_{2}$ stand for means of log-transformed expressions in two analyzed groups, $s_{1}^{2}$ and $s_{2}^{2}$ are estimators of variances, and $n_{1}$ and $n_{2}$ denote the numbers of samples in each of the analyzed groups. In case of log-transform data fold change is simply computed as the difference between the means [13]:

$$
f c=\bar{X}_{1}-\bar{X}_{2} .
$$

In spite of the fact that $t$ is a normalized $f c$, with regard to standard deviation, these two measurements have different motivations and lead to different results. Small $\mathrm{p}$-values of Welch's $t$-test allow to point significantly differentially expressed genes, but possibly with small $f c$. Although possibly biologically relevant, such genes may be difficult to validate with qRT-PCR. On the other hand, scientists may be interested in changes of expression level between groups, measured by $f c$ [13], 
but this statistic does not take into account a variability within groups. Thus, a large relative difference may be statistically insignificant. Moreover, there are approaches proposed to analyze sets of related genes (e.g. Subramanian et al. [20]) which benefit from the ranking of genes based on fold change regardless of significance of differential expression. Additionally, in the analysis of differentially expressed genes the ranking of calculated $\mathrm{p}$-values or, equivalently, values of $t$ is interesting, while in the analysis of fold change nominal values are interesting. Therefore, these two types of study should be treated differently. In our study, we used Spearman's correlation (rank correlation) to compare values of Welch's $t$-test from MA and qRT-PCR, and Pearson's correlation to compare the respective $f c$ values.

Note that our analysis of agreement between detection of differential expression computed from qRT-PCR and MA results fits to the practice of preparing a list of genes with expected FDR below a given threshold. Indeed, a list of genes with expected $F D R \leq \alpha$ is a list with $\mathrm{p}$-values not greater then some $\hat{\alpha}$ dependent on $\alpha$ (this fact results from a characterization of FDR by qvalues - for details see [21]). Hence, the more similar the lists of genes computed from MA and qRT-PCR results are, the higher a rank correlation between $\mathrm{p}$ values is.

To sum up, we analyzed the influence of:

- type of MA study (fold change and differential expression);

- version of annotation of probe sets to genes (new and old);

- type of preprocessing algorithm (MAS5, PLIER, GC-RMA, RMA, MBEI, and MBEImm);

- type of filtering/mapping ( $A, F, D, N, F D, F N)$;

on the correlation between MA and qRT-PCR results.

\section{Results and Discussion \\ Collected data sets}

We collected publications describing both, the MA based gene expression profiling and validation performed on the same RNA samples with qRT-PCR experiments. Next, we asked authors to provide us with raw data (if not publicly available), i.e. microarray CEL files and tables of a threshold cycle $\left(C_{T}\right)$ from qRT-PCR. In this way, we obtained 6 data sets collected in different laboratories. The data sets are listed and abbreviated as follows:

- NerdErd - Samples from patients with non-erosive or erosive reflux disease [22]. We selected 2 groups which were well balanced in respect of sex and the batch effect.
- Strain - Data come from the study of effects of morphine administrations in 4 inbred mice strains [23]. We selected the contrast between 129P3/J and C57BL/6 strains after morphine, because it displays the greatest differences among 4 analyzed strains.

- AgeWT - Age difference (young/old) among wildtype mice. This is one of the most significant contrasts from an unbalanced $2 \times 2$ factorial design study [13] and was analyzed there in detail. We chose this contrast to facilitate comparison with the original study.

- Lonely - Samples of peripheral blood from human individuals differing in the degree of social interactions (high- and low-lonely) used in [12].

- Tsc - Brain specimens, consisting of 4 tumour samples from patients with tuberous sclerosis complex (Tsc) and 3 control samples. The data were performed in the Nencki Institute of Experimental Biology [24], and the MA can be obtained from ArrayExpress [25] (accession number E-MEXP-2351) while additional file 1 contains qRT-PCR results.

- Maqc_CD - In the MAQC project [26] gene expression levels were measured from 2 high-quality, commercially available RNA sample types $A$ and $B$ (Universal Human Reference RNA (UHRR) from Stratagene, Human Brain Reference RNA (HBRR) from Ambion). However, the authors noticed that in practical applications the expected differences between sample types were usually much smaller, and they suggested that mixtures $C$ and $D$ of the original samples were more realistic substitutes of biological samples (page 1157). We followed this suggestion.

For the NerdErd [22], Strain [23], AgeWT [12], and $M a q c_{-} C D$ [26] data sets, we analyzed only one contrast selected from the original multifactorial data. Using the data for all possible contrasts would upset the balance between the experiments in the final average towards the data sets with more groups. Our work, to our knowledge, is the first validation of preprocessing algorithms and filtering/mapping methods on independent data sets collected to address biological questions.

Table 1 presents more details about all described data sets. Let us notice that:

- The analyzed samples originate from different species and were hybridized on different MA types. This variety allows us to assume that the obtained average performance of different ways of MA data analysis is more representative for practical applications.

- Different qRT-PCR protocols were used to validate MA results in different experiments. However, Arikawa 
Table 1 Summary of experiments

\begin{tabular}{lccll}
\hline & Experiment & & Microarrays & qRT-PCR \\
\hline name & ref & \#samp & type & protocol \\
\hline NerdErd & {$[22]$} & 38 & hgu133a2 & SybrGreen \\
Strain & {$[23]$} & 36 & mouse430_2 & TaqMan \\
AgeWT & {$[13]$} & 11 & mgu74av2 & TaqMan \\
Lonely & {$[12]$} & 14 & hgu133a & TaqMan \\
TsC & {$[24]$} & 7 & hgu133plus2 & SybrGreen \\
MaqC_CD & {$[26]$} & 8 & hgu133plus2 & TaqMan \\
\hline
\end{tabular}

The first three columns contain general information about collected experiments: labels of the data sets used in our analysis (name), reference numbers (ref), and numbers of used samples (\#samp). The fourth column describes a type of microarray used in each experiment: (type). The last column contains information about qRT-PCR protocol.

et al. [27] demonstrated that both SYBR Green and TaqMan delivered highly comparable results and both showed high agreement with MA data.

- The collected qRT-PCR data are labelled with gene rather than transcript names and we were not able to gather details of the primers used. Therefore, to compare MA and qRT-PCR results, we had to use annotation of probe sets to genes. In all variants of filtering/mapping except for $D$ and $F D$, several different probe sets may be annotated to one gene. Therefore, it is necessary to transform expression measurements from probe set ensembles to genes. In this study we considered two such transformations: (i) assigning the expression measure from the best probe set (a probe set with the smallest p-value of the Welch's $t$-test) to a gene and (ii) averaging expression measurements over all probe sets annotated to the same gene. It turned out that choosing the best probe set led to consistently higher correlations between MA and qRT-PCR results (both $t$ and $f_{c}$ ), so we present only the results obtained with this method (for details see Methods section and additional file 2).

For all experiments and in each variant of analysis, a number of correlated genes depended on the version of annotations. Table 2 shows a number of genes

Table 2 Number of correlated genes

\begin{tabular}{lllllll}
\hline & NerdErd & Strain & AgeWT & Lonely & Tsc & Maqc_CD \\
\hline A & $8(8)$ & $9(9)$ & $33(35)$ & $6(6)$ & $11(10)$ & $859(876)$ \\
N & $8(7)$ & $9(9)$ & $33(31)$ & $6(6)$ & $11(10)$ & $845(793)$ \\
D & $8(8)$ & $8(8)$ & $28(33)$ & $6(6)$ & $10(10)$ & $835(838)$ \\
F & $8(8)$ & $9(9)$ & $33(35)$ & $5(5)$ & $7(6)$ & $710(731)$ \\
FN & $8(7)$ & $9(9)$ & $33(31)$ & $5(5)$ & $7(6)$ & $697(645)$ \\
FD & $7(7)$ & $8(8)$ & $27(32)$ & $5(5)$ & $6(6)$ & $669(669)$ \\
\hline
\end{tabular}

Number of correlated genes in each variant of filtering/mapping (rows) and a biological experiment (columns). In each cell, the first number stands for the new annotation, whereas the number in brackets stands for the old annotation. correlated in each variant and experiment. In each cell, the first number stands for the new annotation, whereas the number in brackets stands for the old annotation.

\section{Detection of differentially expressed genes}

For every gene validated with qRT-PCR, we calculated Welch's $t$-statistic, both from MA expression measurements and from $\Delta C_{T}$ of qRT-PCR in a given experiment. The two resulting vectors of $t$-statistics (MA and $\Delta C_{T}$ ) were compared by calculating Spearman's correlation. For reasons of simplicity, since high $\Delta C_{T}$ values correspond to low microarray expressions, we multiplied correlations by -1 in figures, and by -100 in tables.

Figure 1 shows average correlations for each of the 6 preprocessing algorithms over the 6 data sets in each of 6 filtering/mapping methods. Plot $1 \mathrm{~A}$ and $1 \mathrm{~B}$ present results for the old and new annotations, respectively. Overall, we observed that using the new annotation led to higher correlations, and the highest correlations were achieved in $F$ and $F N$. For the old annotation a positive interaction between $N$ and $F$ was seen. However for the new annotation naive mapping had almost no influence, therefore, the correlations in $F$ and $F N$ were very similar. Effects of $D$ strongly depended on preprocessing algorithms and, in comparison to $N$, did not show general improvement. Table 3 presents numerical values of the average correlations depicted on Figure 1 and their standard deviations (sd). We can see that GC-RMA, PLIER, and MBEImm outperform RMA, MAS5, and MBEI. Moreover, GC-RMA and PLIER achieved the highest correlations with qRT-PCR in the variants F and FN. On the other hand, MBEImm results are the most correlated with qRT-PCR in the variant FD, but sd of this method is significantly higher then sd of GC-RMA and PLIER.

Figure 2 (parts A, C and E) shows correlations of 3 the best preprocessing algorithms for individual data sets in the variants $A$ and $F$ for both annotations. Let us notice that, for a given annotation, correlations in $F$ are not lower than in $A$. Moreover, the highest correlations were achieved in $F$ and the new annotation in all but one experiment (PLIER in Strain data).

\section{Estimation of the fold change}

Similarly as for detection of differential expression, we compared vectors of MA and qRT-PCR fold changes, but this time we used Pearson's correlations. Figure 3 presents results in a similar way to Figure 1. Overall, we can see that for each preprocessing algorithm and for both annotations correlations are higher than for the differential expression study. Again, the use of the new annotation generally leads to higher correlations. Moreover, for each preprocessing algorithm, the highest correlations were achieved in $F N$ for the old annotation, and in $F$ and $F N$ for the new annotation. However, for 


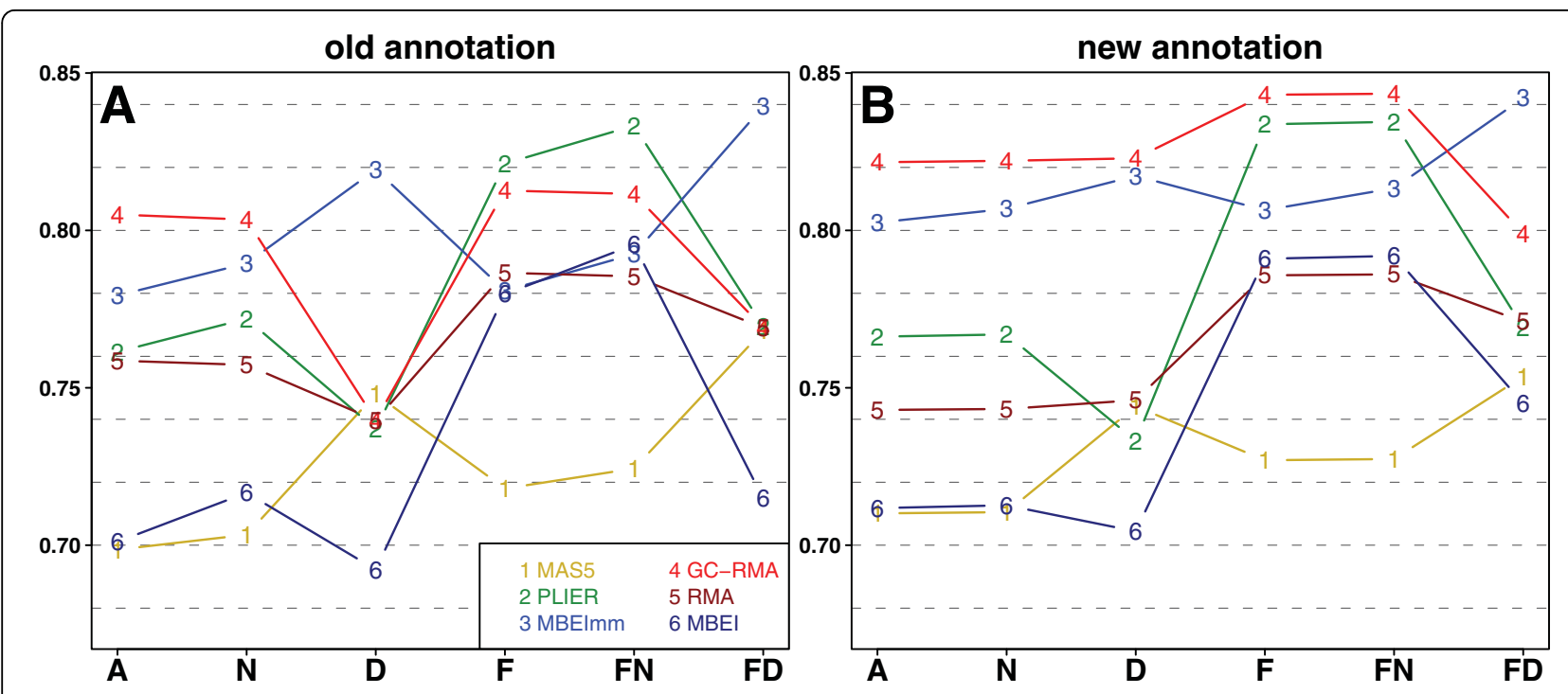

Figure 1 Average Spearman's correlations between MA and qRT-PCR detection of differentially expressed genes. Plots present average correlations between values of Welch's $t$-statistic calculated from MA and GRT-PCR measurements. Average values were calculated for each of the 6 preprocessing algorithms over the 6 data sets in each of the 6 filtering/mapping methods. Panel A shows results for the old annotation while panel B presents results for the new annotation. Curves are coloured and numbered to indicate the preprocessing algorithms.

the new annotation the influence of naive mapping was even smaller than in our differential expression study. The redefinitions $D$ did not have equal influence on all preprocessing algorithms. For both annotations, $D$ led to higher correlations, relatively to $N$, for GC-RMA, RMA, and MAS5, and to lower correlations for PLIER, MBEI, and MBEImm. Table 4 presents average correlations shown in Figure 3 and their standard deviations (sd). Like above, the highest correlations were achieved by PLIER in the variants F and FN. Moreover, the PLIER correlations again have the lowest sd. In general, PM-
MM methods, outperformed PM-only methods regardless of annotations.

Figure 2 (parts B, D, and F) presents correlations of 3 the best preprocessing algorithms for each experiment in the variants $A$ and $F$, for both annotations. As before, correlations in $F$ were not lower than in $A$, and the highest correlations were achieved in $F$ and the new annotation.

\section{Unreliability of results for MBEImm}

Although MBEImm leads to one of 2 the best results, both for detection of differentially expressed genes and

Table 3 Average Spearman's correlations between MA and qRT-PCR detection of differentially expressed genes

\begin{tabular}{|c|c|c|c|c|c|c|c|}
\hline & & MAS5 & PLIER & MBEImm & GC-RMA & RMA & MBEI \\
\hline \multirow[t]{6}{*}{ new annotation } & $A$ & $71 \pm 19$ & $77 \pm 9$ & $80 \pm 14$ & $82 \pm 9$ & $74 \pm 17$ & $71 \pm 15$ \\
\hline & $N$ & $71 \pm 19$ & $77 \pm 9$ & $81 \pm 14$ & $82 \pm 9$ & $74 \pm 17$ & $71 \pm 15$ \\
\hline & $D$ & $74 \pm 8$ & $73 \pm 15$ & $82 \pm 10$ & $82 \pm 8$ & $75 \pm 13$ & $70 \pm 13$ \\
\hline & $F$ & $73 \pm 20$ & $83 \pm 9$ & $81 \pm 13$ & $84 \pm 10$ & $79 \pm 19$ & $79 \pm 13$ \\
\hline & $F N$ & $73 \pm 20$ & $83 \pm 10$ & $81 \pm 14$ & $84 \pm 10$ & $79 \pm 19$ & $79 \pm 13$ \\
\hline & $F D$ & $75 \pm 15$ & $77 \pm 15$ & $84 \pm 13$ & $80 \pm 11$ & $77 \pm 16$ & $74 \pm 10$ \\
\hline \multirow[t]{6}{*}{ old annotation } & $A$ & $70 \pm 20$ & $76 \pm 11$ & $78 \pm 16$ & $81 \pm 12$ & $76 \pm 14$ & $70 \pm 15$ \\
\hline & $N$ & $70 \pm 21$ & $77 \pm 11$ & $79 \pm 15$ & $80 \pm 14$ & $76 \pm 15$ & $72 \pm 15$ \\
\hline & $D$ & $75 \pm 8$ & $74 \pm 14$ & $82 \pm 11$ & $74 \pm 14$ & $74 \pm 14$ & $69 \pm 15$ \\
\hline & $F$ & $72 \pm 22$ & $82 \pm 10$ & $78 \pm 15$ & $81 \pm 13$ & $79 \pm 14$ & $78 \pm 16$ \\
\hline & $F N$ & $72 \pm 22$ & $83 \pm 9$ & $79 \pm 14$ & $81 \pm 15$ & $79 \pm 15$ & $80 \pm 15$ \\
\hline & $F D$ & $77 \pm 13$ & $77 \pm 15$ & $84 \pm 14$ & $77 \pm 16$ & $77 \pm 16$ & $71 \pm 13$ \\
\hline
\end{tabular}

The table contains mean values and the corresponding standard deviations of Spearman's correlation coefficients calculated for 6 experiments. Correlations were computed between values of Welch's $t$-statistic calculated from MA and qRT-PCR measurements. Columns contain results for the 6 preprocessing algorithms, whereas rows contain results for the 6 filtering/mapping methods (negative signs are removed and values are multiplied by 100 ) after the new and the old annotations. 


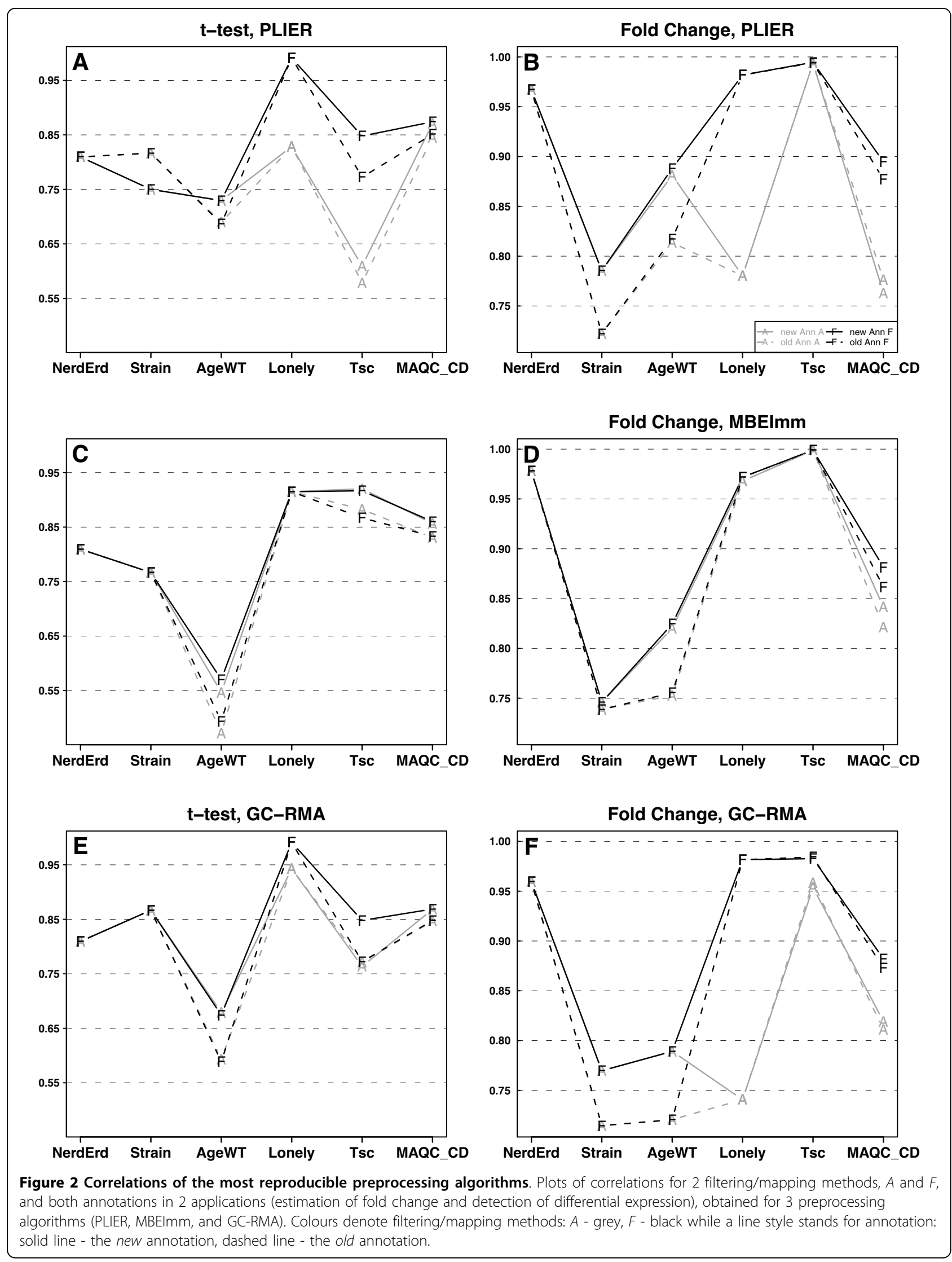




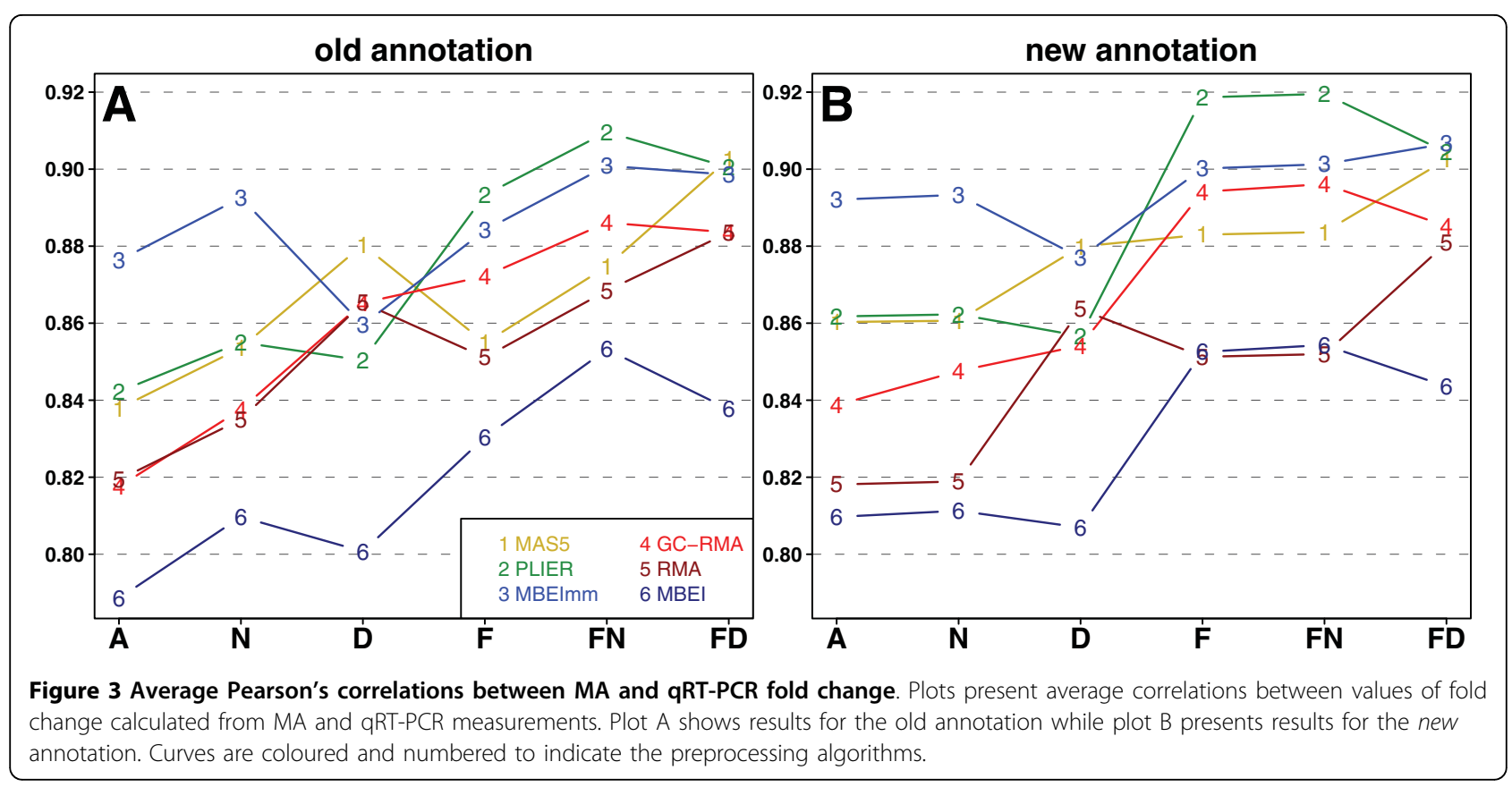

for estimation of the fold change, there are some reasons that undermine this method. Raw MBEImm values may be negative and cannot be log-transformed (such observations are omitted by default). If this occurs for the best probe sets (see the Methods section), then the values of $t$-statistic or fold change are computed using lower number of observations than in case of other preprocessing algorithms or qRT-PCR. For example, Figure (2B, D and $2 \mathrm{~F}$ ) shows that MBEImm clearly outperformed GC-RMA and PLIER in A and N filtering/mapping variants for Lonely data. This result implies a similar order of preprocessing algorithms as in Figure 3, but, as we checked, the values of $f c$ and $\mathrm{t}$ for MBEImm were computed using a lower number of observations than in case of the other preprocessing algorithm. Thus, correlation of MBEImm, and qRT-PCR should not be compared with other preprocessing algorithms. Moreover, in Tables 3 and 4 we show that MBEImm results are less stable (greater sd) than for PLIER or GC-RMA.

\section{Principal Component Analysis of preprocessing algorithms}

Comparison of Table 3 and Table 4 suggests that there is a difference in performance of PM-MM and PM-only methods. In the FN variant of filtering/mapping (generally the best one) using MM probes increased

Table 4 Average Pearson's correlations between MA and qRT-PCR for estimation of the fold change

\begin{tabular}{|c|c|c|c|c|c|c|c|}
\hline & & MAS5 & PLIER & MBEImm & GC-RMA & RMA & MBEI \\
\hline \multirow[t]{6}{*}{ new annotation } & $A$ & $86 \pm 11$ & $86 \pm 10$ & $89 \pm 10$ & $84 \pm 9$ & $82 \pm 14$ & $81 \pm 13$ \\
\hline & $N$ & $86 \pm 11$ & $86 \pm 10$ & $89 \pm 10$ & $85 \pm 10$ & $82 \pm 14$ & $81 \pm 13$ \\
\hline & $D$ & $88 \pm 9$ & $86 \pm 10$ & $88 \pm 10$ & $85 \pm 7$ & $86 \pm 9$ & $81 \pm 14$ \\
\hline & $F$ & $88 \pm 11$ & $92 \pm 8$ & $90 \pm 10$ & $89 \pm 10$ & $85 \pm 15$ & $85 \pm 14$ \\
\hline & $F N$ & $88 \pm 11$ & $92 \pm 8$ & $90 \pm 10$ & $90 \pm 10$ & $85 \pm 15$ & $85 \pm 14$ \\
\hline & $F D$ & $90 \pm 9$ & $90 \pm 9$ & $91 \pm 11$ & $89 \pm 6$ & $88 \pm 9$ & $84 \pm 15$ \\
\hline \multirow[t]{6}{*}{ old annotation } & $A$ & $84 \pm 13$ & $84 \pm 11$ & $88 \pm 12$ & $82 \pm 11$ & $82 \pm 13$ & $79 \pm 15$ \\
\hline & $N$ & $85 \pm 13$ & $86 \pm 11$ & $89 \pm 10$ & $84 \pm 11$ & $84 \pm 11$ & $81 \pm 13$ \\
\hline & $D$ & $88 \pm 9$ & $85 \pm 10$ & $86 \pm 11$ & $87 \pm 9$ & $87 \pm 9$ & $80 \pm 14$ \\
\hline & $F$ & $85 \pm 13$ & $89 \pm 11$ & $88 \pm 12$ & $87 \pm 13$ & $85 \pm 14$ & $83 \pm 17$ \\
\hline & $F N$ & $87 \pm 13$ & $91 \pm 10$ & $90 \pm 10$ & $89 \pm 11$ & $87 \pm 12$ & $85 \pm 14$ \\
\hline & $F D$ & $90 \pm 9$ & $90 \pm 9$ & $90 \pm 12$ & $88 \pm 9$ & $88 \pm 9$ & $84 \pm 16$ \\
\hline
\end{tabular}

The table contains mean values and the corresponding standard deviations of Pearson's correlation coefficients. Correlations were computed between fold changes calculated from MA and qRT-PCR measurements. Columns contain results for the 6 preprocessing algorithms, whereas rows contain results for the 6 filtering/mapping methods (negative signs are removed and values are multiplied by 100) after the new and the old annotations. 
correlation in the fold change studies, but PM-only methods performed better in the detection of significant expression. We confirmed the difference in performance of PM-MM and PM-only preprocessing algorithms in Principal Component Analysis on MAQC data. We used $A$ and $B$ sample types, instead of $C$ and $D$ because we wanted to obtain the maximal biological diversity and to analyze only technical differences. We performed PCA on values of $t$-test and fold change separately using only genes that were validated with qRT-PCR. Then, we plotted the preprocessing algorithms on the plane of the first $(\mathrm{PC} 1)$ and the second ( $\mathrm{PC} 2)$ principal directions. Figure 4 presents both results.

The relevance of PC1 and PC2 measured with a sum of relative variances of first two PC amounts to $77 \%$ for detection of differentially expressed genes, and to $83 \%$ for estimation of fold change. Discrimination between PM-only PM-MM methods is well noticeable, especially in Figures 4A, where PC1 separates PM-only from PMMM methods. Additionally, in both pictures, the PMMM algorithms are placed more closely to each other. In both cases, MBEI and MBEImm algorithms are separated by the first principal component.

\section{Conclusions}

This paper presents a comparison of preprocessing algorithms and filtering/mapping variants for analysis of microarray data. Based on 6 independent biological data sets, we studied correlations between MA and qRT-PCR expression measurements in 2 studies (detection of differentially expressed genes and estimation of fold change) using 2 annotations (new and old). We showed that filtering by fraction Present calls increased correlations for all 6 preprocessing algorithms. We observed the difference in performance of PM-MM and PM-only methods: using MM probes increased correlations in the fold change studies, but PM-only methods performed better in the detection of significant expression. For detection of differentially expressed genes, we recommend using GC-RMA, and for estimation of fold change - PLIER algorithm. The use of the more recent annotation improves results in both types of studies, encouraging re-analysis of old data.

\section{Methods}

RNA assays

As the NerdErd, Strain, AgeWT, and Lonely data were taken from already published papers, we describe only the generation of the Tsc data. Total RNA was prepared by Tri-Reagent (Sigma) extraction from snap-frozen tissues and cleaned up using RNeasy Mini Kit according to the manufacturer's protocol (Qiagen). The quality and quantity of RNA were verified using the Agilent bioanalyzer (Agilent Technologies). All the microarray preparation procedures were done according to recommendations of Affymetrix (Santa Clara, CA) using $5 \mu \mathrm{g}$ of total RNA as a template. Fragmented cRNA was hybridized first to a control microarray (Test3) and then, after sample quality evaluation, to the HG-U133 Plus 2.0 arrays (Affymetrix). Samples hybridization was done in the Department of Nuclear Medicine and Endocrine Oncology, Maria Sklodowska-Curie Memorial

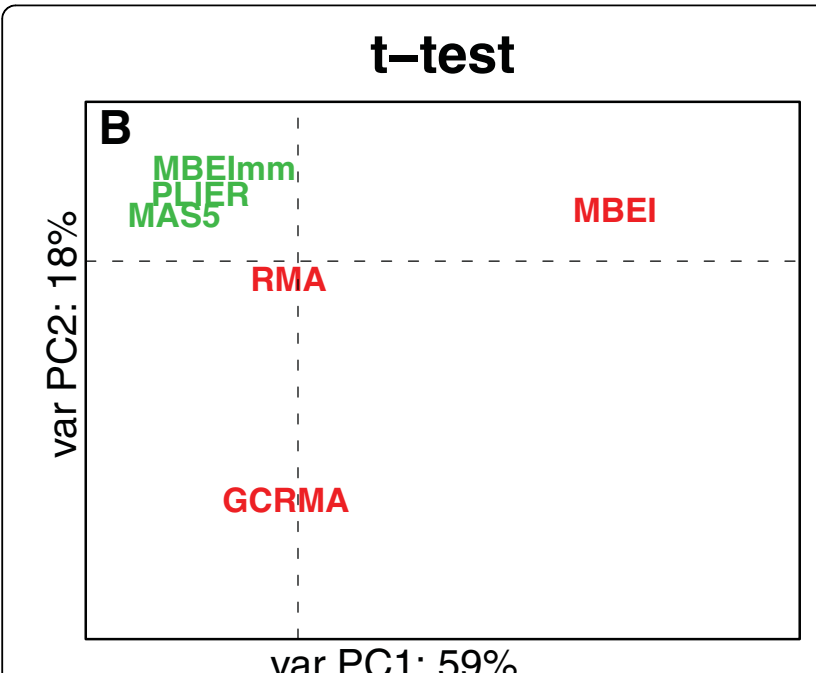

Fold Change

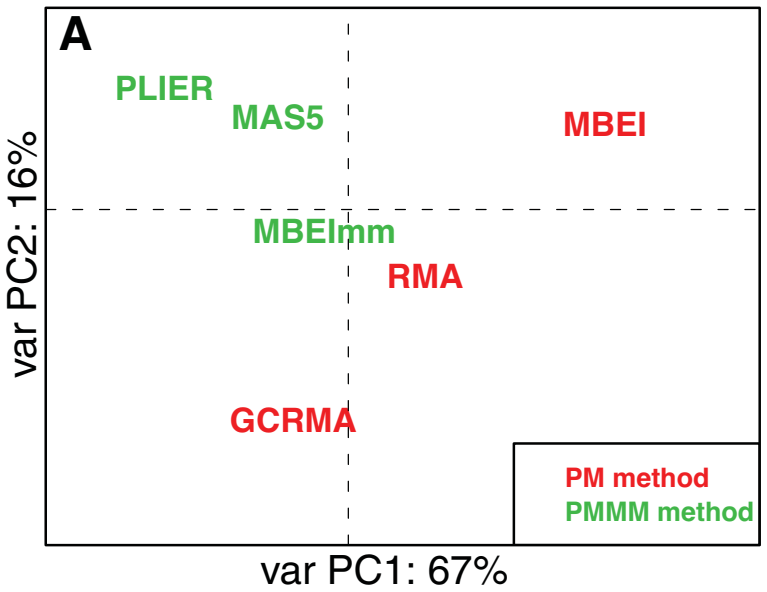

Figure 4 Principal Component Analysis of preprocessing algorithms in variant A. Plots present Principal Component Analysis of preprocessing algorithms on sample types A and B from MAQC study. Algorithms using the MM probes are marked green while the PM-only algorithms are marked red. (A) PCA of values of Welch's $t$-statistic from microarray and qRT-PCR detection of differentially expressed genes. (B) PCA of values of fold change between groups. In both cases the new annotation was used. Axes are labelled with percentage of total variability explained by PC1 and PC2. It should be noted that in this technical comparison we used samples of original RNA instead of mixtures $\mathrm{C}$ and D. 
Cancer Center and Institute of Oncology, Gliwice, Poland. The cDNAs were synthesized by extension of oligo $(d T)_{15}$ primers with 200 units of M-MLV reverse transcriptase (Sigma) in a mixture containing $1 \mu \mathrm{g}$ of total RNA in $20 \mu \mathrm{l}$. Real-time PCR analysis was performed in duplicate using the 7500 Real Time PCR System (Applied Biosystems) on cDNA equivalent to $10 \mathrm{ng}$ RNA in $20 \mu \mathrm{l}$ reaction volume containing $1 \times$ SYBR Green PCR master mix (Applied Biosystems) and the primer sets QuantiTect Primer Assays (200) (Qiagen). 18SrRNA was used as an internal control with primers designed by the Primer Express Software (Applied Biosystems).

\section{Filtering by fraction Present calls}

$\mathrm{P} / \mathrm{A} / \mathrm{M}$ detection algorithm was originally designed for a single array analysis to verify if a particular probe set measured specific or non-specific hybridization signal. This algorithm is based on Wilcoxon signed rank test and was introduced by Affymetrix [6]. Each analyzed probe set gets one of three possible labels depending on signals of PM and MM probes. Label P (Present) means that the signal is specific, while label A (Absent) stands for the lack of specific signal. The third label, M (Marginal), means that specificity is not clear.

We implemented one variant of filtering by fraction Present calls proposed in [14]. We removed probe sets which got less than $25 \%$ percent of calls $\mathrm{P}$ in the analyzed experiments. Only for the $T s c$, we decided to raise the threshold to $50 \%$, because of a small number of samples. A similar approach, but without using information about the treatment assignments, was used in [15].

\section{Naive mapping}

Naive mapping consists in exclusion of all probe sets that are annotated to more than one gene (one-tomany). To do so, we used annotations of probe sets to genes provided in the Ensembl database (identifiers with ENSG prefix in human or ENSMUSG prefix in mice) [28].

\section{Calculation of gene expression from probe sets annotated to the same gene}

In probe sets to gene annotation, it may be observed that (i) only one probe set is annotated to a given gene or (ii) several probe sets are annotated to the same gene (annotation of one probe set to more that one gene is a filtering/mapping problem). In the first case, we simply used the received signal and in the second case, we used the best probe set (probe sets with the smaller $\mathrm{p}$-values of $t$-test among all probe sets annotated to the same gene) to quantify gene $t$-test or fold change. For all analyzed data sets, there were ensembles of probe sets annotated to the same gene irrespective of the filtering/ mapping variants (besides $F D$ and $D$ ).

We also tried to evaluate gene-specific values without consideration of the treatment assignments. For each microarray, we computed an arithmetic mean as well as a Tukey biweight. Both ways of averaging led to similar results and were outperformed by the "best probe set" method. In Additional file 2, we present results only for an arithmetic mean, because it is easier and faster to compute it than a Tukey biweight function.

\section{Preprocessing of microarray data}

All calculations were performed in R statistical environment [29] and relevant Bioconductor software [30]. The mas5( ), rma( ), and gcrma( ) functions were used with default parameters. To apply MBEI and MBEImm, we used expresso( ) function according to description in [31]. To apply PLIER, we used justPLIER( ) function from plier package with a normalize parameter set on TRUE according to recommendation in [7]. Before further analysis, we log-transformed the results of MAS5, MBEImm, and MBEI. The example of used $R$ code is introduced in Additional file 3.

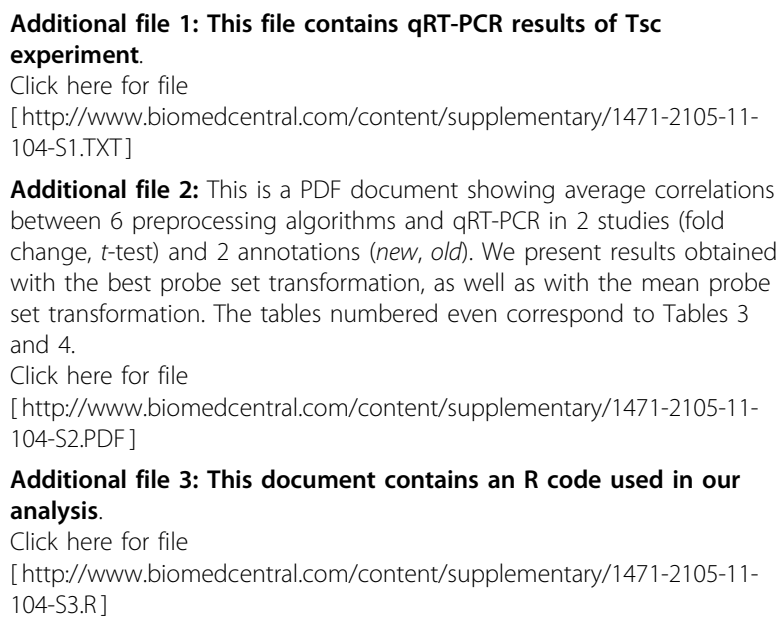

Additional file 2: This is a PDF document showing average correlations between 6 preprocessing algorithms and qRT-PCR in 2 studies (fold

change, $t$-test) and 2 annotations (new, old). We present results obtained with the best probe set transformation, as well as with the mean probe set transformation. The tables numbered even correspond to Tables 3 and 4 .

Click here for file

[http://www.biomedcentral.com/content/supplementary/1471-2105-11104-S2.PDF ]

Additional file 3: This document contains an $\mathrm{R}$ code used in our analysis.

Click here for file

[http://www.biomedcentral.com/content/supplementary/1471-2105-11104-S3.R ]

\section{Acknowledgements}

We thank S. Cole, K. F. Kerr, J. Ostrowski, M. Korostynski and M. Piechota for the permission to use their data. This work was supported by grant PBZMNil-2/1/2005 from the Ministry of Science and Higher Education (Poland)

\section{Author details}

'Laboratory of Transcription Regulation, Department of Cell Biology, The Nencki Institute of Experimental Biology, Pasteur 3, $02-093$ Warsaw, Poland. ${ }^{2}$ Institute of Informatics, Faculty of Mathematics, Informatics and Mechanics, Banacha 2, University of Warsaw, 02-097 Warsaw, Poland.

\section{Authors' contributions}

$J M$ and PP designed the study and wrote R scripts. MD provided biological expertise to the microarrays technology and the Ensembl annotation. MT prepared all RNA and performed the GRT-PCR experiment for the Tsc data set. JM performed the statistical analysis and drafted the manuscript. JM, PP, 
MT and MD wrote the manuscript. All authors read and approved the final manuscript.

\section{Received: 6 July 2009}

Accepted: 24 February 2010 Published: 24 February 2010

\section{References}

1. Shedden K, Chen W, Kuick R, Ghosh D, MacDonald J, Cho KR, Giordano TJ, Gruber SB, Fearon ER, Taylor JMG, Hanash S: Comparison of seven methods for producing Affymetrix expression scores based on False Discovery Rates in disease profiling data. BMC Bioinformatics 2005, 6:26.

2. Zakharkin SO, Kim K, Mehta T, Chen L, Barnes S, Scheirer KE, Parrish RS, Allison DB, Page GP: Sources of variation in Affymetrix microarray experiments. BMC Bioinformatics 2005, 6:214.

3. Millenaar FF, Okyere J, May ST, van Zanten M, Voesenek LA, Peeters AJ: How to decide? Different methods of calculating gene expression from short oligonucleotide array data will give different results. $B M C$ Bioinformatics 2006, 7:137.

4. Seo J, Hoffman EP: Probe set algorithms: Is there a rational best bet? BMC Bioinformatics 2006, 7:395.

5. Irizarry RA, Wu Z, Jaffee $\mathrm{H}$ : Comparison of Affymetrix GeneChip expression measures. Bioinformatics 2006, 22:789-794.

6. Affymetrix Inc: Statistical algorithms description document. Tech rep 2002http://www.affymetrix.com/support/technical/whitepapers/ sadd_whitepaper.pdf.

7. Affymetrix Inc: Guide to Probe Logarithmic Intensity Error (PLIER) Estimation. Tech rep 2007http://www.affymetrix.com/support/technical/ technotes/plier technote.pdf

8. Irizarry RA, Bolstad BM, Collin F, Cope LM, Hobbs B, Speed TP: Summaries of Affymetrix GeneChip probe level data. Nucleic Acids Res 2003, 31:e15.

9. Wu Z, Irizarry RA: Preprocessing of oligonucleotide array data. Nat Biotechnol 2004, 22:656-658.

10. Li C, Wong WH: Model-based analysis of oligonucleotide arrays: model validation, design issues and standard error application. Genome Biol 2001, 2:research0032.

11. Li C, Wong WH: Model-based analysis of oligonucleotide arrays: Expression index computation and outlier detection. Proc Natl Acad Sci USA 2001, 98:31-36.

12. Cole S, Hawkley L, Arevalo J, Sung C, Rose R, Cacioppo J: Social regulation of gene expression in human leukocytes. Genome Biol 2007, 8:R189,

13. Qin LX, Beyer RP, Hudson FN, Linford NJ, Morris DE, Kerr KF: Evaluation of methods for oligonucleotide array data via quantitative real-time PCR. BMC Bioinformatics 2006, 7 .

14. McClintick JN, Edenberg HJ: Effects of filtering by Present call on analysis of microarray experiments. BMC Bioinformatics 2006, 7:49.

15. Hackstadt A, Hess A: Filtering for increased power for microarray data analysis. BMC Bioinformatics 2009, 10:11.

16. Okoniewski MJ, Miller CJ: Hybridization interactions between probesets in short oligo microarrays lead to spurious correlations. BMC Bioinformatics 2006, 7:276.

17. Dai M, Wang P, Boyd A, Kostov G, Athey B, Jones E, Bunney W, Myers R, Speed T, Akil H, Watson $S$, Meng F: Evolving gene/transcript definitions significantly alter the interpretation of GeneChip data. Nucleic Acids Res 2005, 33:e175.

18. Sandberg R, Larsson O: Improved precision and accuracy for microarrays using updated probe set definitions. BMC Bioinformatics 2007, 8:48.

19. Jiang $N$, Leach $L$, Hu X, Potokina E, Jia T, Druka A, Waugh R, Kearsey MJ, Luo Z: Methods for evaluating gene expression from Affymetrix microarray datasets. BMC Bioinformatics 2008, 9:284.

20. Subramanian A, Tamayo P, Mootha V, Mukherjee S, Ebert B, Gillette M, Paulovich A, Pomeroy S, Golub T, Lander E, Mesirov J: Gene set enrichment analysis: A knowledge-based approach for interpreting genome-wide expression profiles. Proc Natl Acad Sci USA 2005, 102(43): 15545

21. Storey J, Tibshirani R: Statistical significance for genome-wide studies. Proc Natl Acad Sci 2003, 100:9440.

22. Ostrowski J, Rubel T, Wyrwicz LS, Mikula M, Bielasik A, Butruk E, Regula J: Three clinical variants of gastroesophageal reflux disease form two distinct gene expression signatures. J Mol Med 2006, 84:872-882.

23. Korostynski M, Piechota M, Kaminska D, Solecki W, Przewlocki R: Morphine effects on striatal transcriptome in mice. Genome Biol 2007, 8:R128.
24. Tyburczy ME, Kotulska K, Pokarowski P, Mieczkowski J, Kucharska J, Grajkowska W, Roszkowski M, Jozwiak S, Kaminska B: Novel proteins regulated by mTOR in subependymal giant cell astrocytomas of patients with tuberous sclerosis complex and new therapeutic implications. Am J Pathol.

25. Parkinson H, Sarkans U, Shojatalab M, Abeygunawardena N, Contrino S, Coulson R, Farne A, Lara G, Holloway E, Kapushesky M, Lilja P, Mukherjee G, Oezcimen A, Rayner T, Rocca-Serra P, Sharma A, Sansone S, Brazma A: ArrayExpress - a public repository for microarray gene expression data at the EBI. Nucleic Acids Res 2005, , 33 Database: D553-5.

26. Shi L, Reid LH, Jones WD, Shippy R, Warrington JA, Baker SC, Collins PJ, de Longueville F, Kawasaki ES, Lee KY, Luo Y, Sun YA, Willey JC, Setterquist RA, Fischer GM, Tong W, Dragan YP, Dix DJ, Frueh FW, Goodsaid FM, Herman D, Jensen RV, Johnson CD, Lobenhofer EK, Puri RK, Scherf U, Thierry-Mieg J, Wang C, Wilson M, Wolber PK, Zhang L, Amur S, Bao W, Barbacioru CC, Lucas AB, Bertholet V, Boysen C, Bromley B, Brown D, Brunner A, Canales R, Cao XM, Cebula TA, Chen JJ, Cheng J, Chu TM, Chudin E, Corson J, Corton JC, Croner LJ, Davies C, Davison TS, Delenstarr G, Deng X, Dorris D, Eklund AC, Fan XH, Fang H, FulmerSmentek S, Fuscoe JC, Gallagher K, Ge W, Guo L, Guo X, Hager J, Haje PK, Han J, Han T, Harbottle HC, Harris SC, Hatchwell E, Hauser CA, Hester S, Hong $H$, Hurban $P$, Jackson SA, Ji H, Knight CR, Kuo WP, Leclerc JE, Levy S, Li QZ, Liu C, Liu Y, Lombardi MJ, Ma Y, Magnuson SR, Magsodi B, McDaniel T, Mei N, Myklebost O, Ning B, Novoradovskaya N, Orr MS, Osborn TW, Papallo A, Patterson TA, Perkins RG, Peters EH, Peterson R, Philips KL, Pine PS, Pusztai L, Qian F, Ren H, Rosen M, Rosenzweig BA, Samaha RR, Schena M, Schroth GP, Shchegrova S, Smith DD, Staedtler F, Su Z, Sun H, Szallasi Z, Tezak Z, Thierry-Mieg D, Thompson KL, Tikhonova I, Turpaz $Y$, Vallanat $B$, Van $C$, Walker $S$ J, Wang $S$ J, Wang $Y$, Wolfinger $R$, Wong A, Wu J, Xiao C, Xie Q, Xu J, Yang W, Zhang L, Zhong S, Zong Y, Slikker W Jr: The MicroArray Quality Control (MAQC) project shows interand intraplatform reproducibility of gene expression measurements. Nat Biotechnol 2006, 24(9):1151-1161.

27. Arikawa E, Sun Y, Wang J, Zhou Q, Ning B, Dial S, Guo L, Yang J: Crossplatform comparison of SYBR(R) Green real-time PCR with TaqMan PCR, microarrays and other gene expression measurement technologies evaluated in the MicroArray Quality Control (MAQC) study. BMC Genomics 2008, 9:328.

28. Hubbard T, Aken B, Ayling S, Ballester B, Beal K, Bragin E, Brent S, Chen Y, Clapham P, Clarke L, Coates G, Fairley S, Fitzgerald S, Fernandez-Banet J, Gordon L, Graf S, Haider S, Hammond M, Holland R, Howe K, Jenkinson A, Johnson N, Kahari A, Keefe D, Keenan S, Kinsella R, Kokocinski F, Kulesha E, Lawson D, Longden I: Ensembl 2009. Nucleic Acids Res 2009, 37:D690-697.

29. R Development Core Team: R: A Language and Environment for Statistical Computing R Foundation for Statistical Computing, Vienna, Austria 2009http://www.R-project.org.

30. Gentleman R, Carey VJ, Bates DM, Bolstad B, Dettling M, Dudoit S, Ellis B, Gautier L, Ge Y, Gentry J, Hornik K, Hothorn T, Huber W, lacus S, Irizarry R, Leisch F, Li C, Maechler M, Rossini AJ, Sawitzki G, Smith C, Smyth G, Tierney L, Yang JYH, Zhang J: Bioconductor: Open software development for computational biology and bioinformatics. Genome Biol 2004, 5:R80.

31. Gentelman R, Irizarry RA, Carey VJ, Dudoit S, Huber W: Preprocessing Highdensity Oligonucleotide Arrays. Bioinformatics and Computational Biology Solutions Using R and Bioconductor New York: Springer, 1 2005, 26.

\section{doi:10.1186/1471-2105-11-104}

Cite this article as: Mieczkowski et al:: Probe set filtering increases correlation between Affymetrix GeneChip and qRT-PCR expression measurements. BMC Bioinformatics 2010 11:104. 